\title{
Sensing Emergence in Complex Systems
}

\author{
Muaz A. Niazi, Senior Member IEEE, and Amir Hussain, Senior Member IEEE
}

\begin{abstract}
We propose the Sensing of Emergent behavior in a Complex Adaptive System (SECAS), an extension of our previous work Formal Agent Based Simulation Framework (FABS). Using aggregated data from an array of proximity sensors, SECAS allows for the detection of complex behavior such as flocking of mobile robots or life forms. For validation, we develop an agentbased simulation model. Extensive simulation experiments using a wide range of randomly deployed sensors demonstrate the effectiveness of SECAS in the sensing of flocking.
\end{abstract}

Index Terms - Event Detection, Multiagent Systems, Agentbased Simulation, Complex Adaptive System, Environment, Flocking, Cognitive Sensor Networks

\section{INTRODUCTION}

$\mathrm{C}$ YOMPLEX Adaptive Systems (cas) are known to exhibit discernable global patterns termed as "emergence"[1]. These patterns emerge as a result of nonlinear interactions of numerous components of the cas. Traditionally, emergent behavior has been considered to be detectable by intelligent observers[2]. With the recent advances in sensors, it seems appropriate to explore how seemingly simple sensors might be useful in the detection of such intelligent behavior.

Agent-based modeling has previously been shown as the prime choice for modeling and simulation of complex and selforganizing systems such as in the domain of sensor-based, P2P and ad-hoc networks [3].

Emergent behavior has traditionally been considered difficult to define and hard to detect. Boschetti and Gray [2] describe three levels of emergence:

1. Pattern Formation and detection such as oscillating reactions etc.

2. Intrinsic Emergence such as flocking behavior

3. Causal Emergence such as human behavior using messaging.

In our previous paper [4], we proposed a Formal Agentbased Simulation Framework (FABS), a formal specification framework coupled with an agent-based model for modeling and simulation of sensing in a complex environment. In FABS,

Copyright $($ IEEE 2011 (Author's version of paper)

Cite as Muaz Niazi and Amir Hussain, Sensing Emergence in Complex Systems, IEEE Sensors Journal (in-press)

Manuscript received March 7, 2011, Revised April 7, 2011, Accepted April 7, 2011.

M. A. Niazi is with the COMSATS Institute of IT, Islamabad, Pakistan and also the University of Stirling, Scotland, UK (Tel: 92-321-5310906; email: man@ cs.stir.ac.uk).

A. Hussain is with the University of Stirling, Scotland, UK. (e-mail: ahu@cs.stir.ac.uk). the goal was to couple the application of an ISO standard formal specification language " $Z$ ' with the development of an agent-based model of sensors. FABS however was neither concerned with empirical results nor specified any rules for sensing emergence. In this letter, we extend FABS by proposing Sensing of Emergence in Complex Adaptive Systems (SECAS). SECAS utilizes aggregate data from a randomly deployed sensor array to sense "emergent" flocking behavior [5] of "boids" in an environment.

The structure of the rest of the paper is as follows:

We first describe formal Methodological development. Next, we discuss the simulation experiments and results. Finally, we conclude the paper.

\section{Methodology}

SECAS is based on the use of a distributed set of sensors to collectively sense a complex behavior. The basic hypothesis can thus be defined as follows:

\section{A. Definition 1:}

Emergent behavior can be perceived, granted if it is associated, firstly, with an environmental change, which can be "sensed" and secondly, the values can somehow be used in an aggregation function reflecting the emergent effect as a manifestation of the global environmental change

In the chosen example of flocking, we note that the complex flocking phenomenon requires the "boids" to come closer while moving in a seemingly unconstrained fashion. Flocking boids come closer to each other without jumbling together. To sense this complex behavior, we note that intuitively, the lower the total number of "proximity" sensors that would turn on (being in the vicinity of boids), the more the emergent flocking behavior would deemed to have occurred. Thus, SECAS rule for the "flocking world" can be stated as following:

\section{B. Definition 2:}

In a contained but unconstrained world with an array of proximity sensors, the emergent flocking behavior of boids can be detected by the examination of aggregation trends in historical data collected from the sensors.

\section{Measurements}

The key measurement in SECAS is "Sensed" (represented by $S(t)$ ), the mathematical aggregation (sum) of measurements of all sensors at any given time $t$. So, every time a sensor turns on, it is considered to add to the Sensed value. Thus, Sensed would always be less than or equal to the

\footnotetext{
${ }^{1}$ Boids being a general term that can be applied to entities ranging from a group of mobile robots to intelligent life forms such as insects, birds or even fish.
} 
total number of available sensors in the world at that instance $\left(N_{s}(t)\right)$ as shown in equation 1.1.

$$
S(t) \leq N_{s}(t)
$$

If $n_{d}(t)$ represents a single sensor which is detecting "boids" in its proximity, from a total of $i$ active sensors at a given time, $S(t)$ being the sum of values of all proximity sensors, turning on, would thus be formally defined as follows:

$$
S(t)=\sum_{d=0}^{d=i} n_{d}(t)
$$

\section{SIMULATION EXPERIMENTS}

In this section, we first discuss the experimental setup for the simulation experiments followed by a discussion of results.

\section{A. Experimental setup}

Following on to previous work on the simulation of flocking behavior, the simulated world is assumed to be "toroidal". Toroidal worlds allow the boids to move in an unconstrained manner while still allowing the sensors to observe them. Sensors are considered to be low-power consuming devices such as those based on [6] and capable of detection of presence of boids e.g. using infra-red sensing. When "boids" come in the vicinity of a sensor, the sensor is visualized with an increase in the size of the sensor as shown in Figure 1.

\section{B. Description of Experiment sets}

To eliminate the possible effects of random behavior, each simulation was executed 10 times. Each individual experiment continued for 1000 steps. Summarized simulation results are subsequently plotted using high-low graphs in a 95\% Confidence Interval.

\section{1) Experiment set I}

In the first experiment set, the number of boids was set as a constant (50) while the number of sensors was varied from 100 to 1000 sensors as shown in Figure 1.

\section{2) Experiment set II}

The second experiment set was a validation exercise. It was used to evaluate the effects of varying the number of boids in the flocking world on the effectiveness of using Sensed as a means of detecting flocking. Here the number of sensors was set as a constant (1000) while the number of boids was varied from 50 to 450 boids.

\section{Discussion of Results}

Results of Experiment set I can be observed in Figure 2. Here, as flocking takes place, gradually the Sensed value is minimized. The effectiveness of SECAS can be seen since similar results can be observed even when sensors are varied from 1000 to 100 sensors, Whereas in Experiment set II varying the number of boids does not have any significant effect on the detection of flocking. Thus, a smaller number of boids in the environment can still be detected using SECAS (by using the Sensed value) with the same precision as a larger number of boids as shown in Figure 3. Thus, empirical results explored by performing numerous experiments demonstrate the effectiveness of SECAS in a variety of scenarios.

\section{CONCLUSIONS AND FUTURE WORK}

In this paper, we have proposed SECAS for the detection of emergent behavior in the vicinity of randomly deployed sensors. Our extensive simulation experiments validate the effectiveness of SECAS in detecting emergent behavior in general and flocking behavior in particular. In the future, we hope to extend SECAS in the domain of other emergent phenomena in various types of cas.

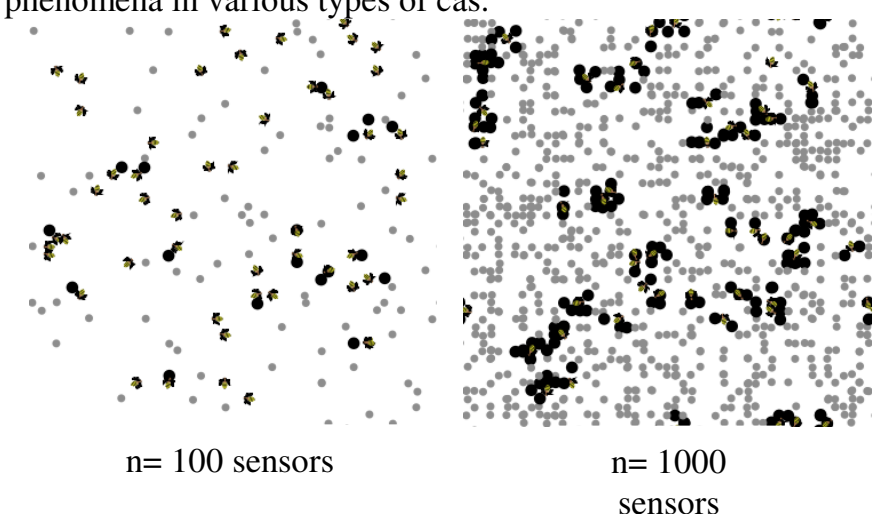

Figure 1 Simulation experiments with varying numbers of sensors

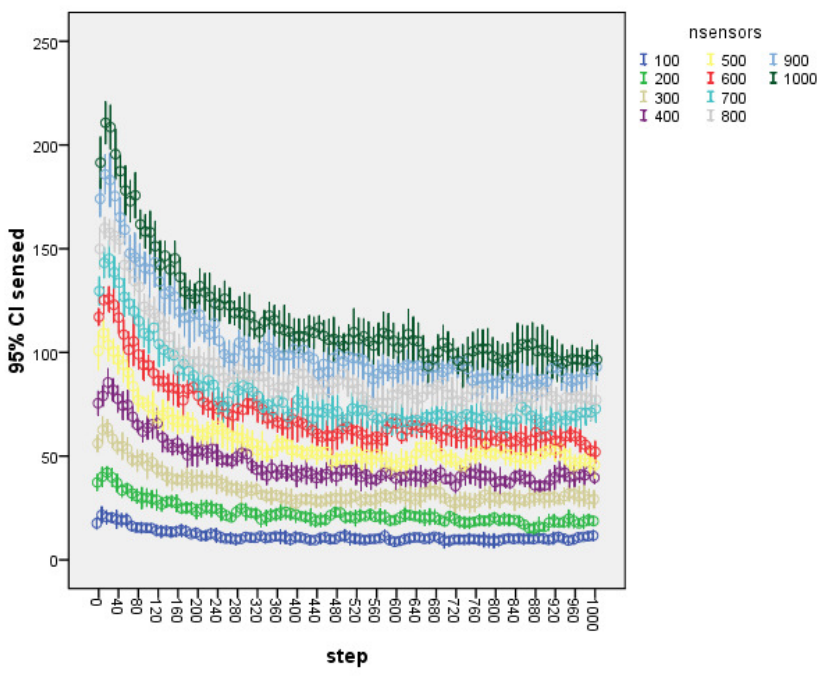

Figure 2 95\% CI based value of sensed 


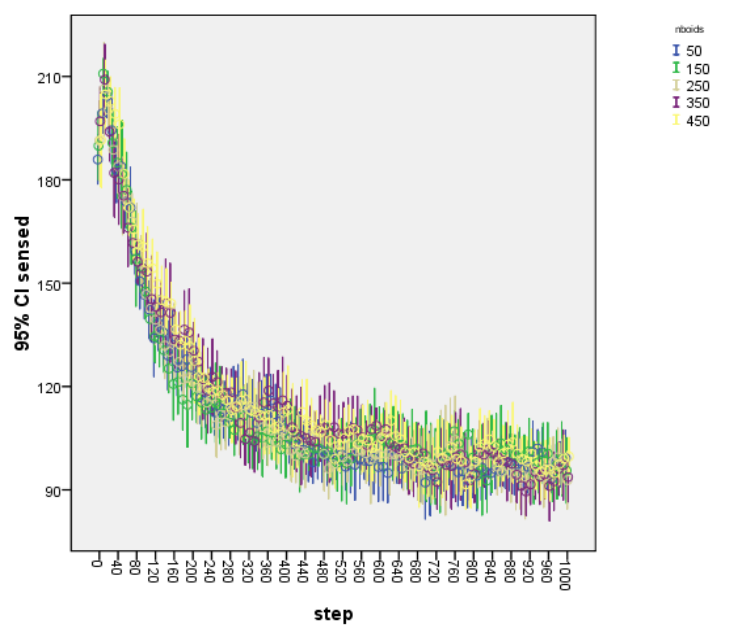

Figure $395 \%$ CI value of sensed while varying the number of boids

\section{REFERENCES}

[1]J. Holland, "Complex adaptive systems," Daedalus, vol. 121, no. 1, pp. 17-30, 1992.

[2]F. Boschetti, and R. Gray, "A Turing Test for Emergence," Advances in Applied Self-organizing Systems, Advanced Information and Knowledge Processing M. Prokopenko, ed., pp. 349-364: Springer London, 2008.

[3]M. Niazi, and A. Hussain, “ Agent based Tools for Modeling and Simulation of Self-Organization in Peer-to-Peer, Ad-Hoc and other Complex Networks," IEEE Communications Magazine, vol. 47, no. 3, pp. 163 - 173., March 2009, 2009.

[4]M. A. Niazi, and A. Hussain, "A Novel Agent-Based Simulation Framework for Sensing in Complex Adaptive Environments," Sensors Journal, IEEE, vol. 11, no. 2, pp. 404-412, 2011.

[5]C. W. Reynolds, "Flocks, herds and schools: A distributed behavioral model," in Proceedings of the 14th annual conference on Computer graphics and interactive techniques, 1987, pp. 25-34.

[6]P. Dutta, M. Grimmer, A. Arora, S. Bibyk, and D. Culler, "Design of a wireless sensor network platform for detecting rare, random, and ephemeral events," in Proceedings of the 4th international symposium on Information processing in sensor networks, Los Angeles, California, 2005, pp. 70. 